\title{
CHARACTERIZATION OF THE INESSENTIAL ENDOMORPHISMS IN THE CATEGORY OF ABELIAN GROUPS
}

\author{
S. Abdelalim and H. Essannouni*
}

Abstract

An endomorphism $f$ of an Abelian group $A$ is said to be inessential (in the category of Abelian groups) if it can be extended to an endomorphism of any Abelian group which contains $A$ as a subgroup. In this paper we show that $f$ is as above if and only if $\left(f-v \operatorname{id}_{A}\right)(A)$ is contained in the maximal divisible subgroup of $A$ for some $v \in \mathbb{Z}$.

\section{Introduction}

Throughout this paper, we will follow the terminology of [2]. Let $M$ be an object of a category $\mathcal{C}$ and $f \in \operatorname{End}(M), f$ is called inessential (in $\mathcal{C}$ ) if for any monomorphism $\sigma: M \rightarrow N$ there exists $\tilde{f} \in \operatorname{End}(N)$ such that $\tilde{f} \sigma=\sigma f$, in other words the following diagram

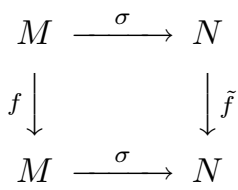

commutes.

Ines $(M)$ denotes all the inessential endomorphisms of $M . M$ is called rigid if $\operatorname{End}(M)=\operatorname{Ines}(M)$. For a concrete category $\mathcal{C}$, the characterization of the inessential endomorphisms is one of the problems raised in [2]. In this paper, we take $\mathcal{C}=A b$ the category of the Abelian groups and we show for an Abelian group $A$, and an endomorphism $f$ of $A$, that $f$ is inessential (in $A b$ ) if and only if there exists $v \in \mathbb{Z}$ such that

2000 Mathematics Subject Classification. 20K30.

Key words. Category, inessential, monomorphism, rigid, divisible, extension.

* The second author is partially supported by the Ministerío de Ciencía y Tecnología. Proyecto BEM 2001-2335, and he thanks Professor A. Kaidi for the helpful conversations during his visit to the University of Almería. 
$\left(f-v \operatorname{id}_{A}\right)(A) \subseteq D$, where $D$ is the maximal divisible subgroup of $A$. In particular if $A$ is reduced then $\operatorname{Ines}(A)=\mathbb{Z} \mathrm{id}_{A}$. The proof of this result uses the properties of the endomorphisms of some extensions of certain direct sums of torsion cyclic groups.

From now on, the word group means Abelian group and we adopt the notations of $[3]$.

\section{Some constructions}

Construction 1. Let $\left(\alpha_{n}\right)_{n \geq 0}$ be a sequence of natural numbers such that $\alpha_{n}<\alpha_{n+1}$ and $2 \alpha_{n+1}-\alpha_{n}+n+3 \leq \alpha_{n+2}, \forall n \in \mathbb{N}$. If we put $\theta_{n}=\alpha_{n}-\alpha_{n-1}-n$ for $n \geq 1$ then we have $\theta_{n}-\theta_{n-1} \geq n, n \geq 2$. Let $p \in \mathbb{N}^{*}$ and $\left(t_{n, m}\right)_{n \geq m}$ be a set of nonzero natural numbers, relatively prime with $p$ such that $t_{i, j} t_{j, k}=t_{i, k}$ if $i \geq j \geq k$.

We consider the direct product $\prod_{n \geq 1}\left\langle x_{n}\right\rangle$ with $o\left(x_{n}\right)=p^{\alpha_{n}}$ and denote by $\varphi_{k}: \prod_{n \geq 1}\left\langle x_{n}\right\rangle \rightarrow\left\langle x_{k}\right\rangle$ the canonical projection. For $m \geq 1$, we define the element $g_{m}$ of $\prod_{n \geq 1}\left\langle x_{n}\right\rangle$ by

$$
\varphi_{n}\left(g_{m}\right)= \begin{cases}0 & \text { if } n<m \\ p^{\alpha_{n}-\alpha_{m}} x_{n} & \text { if } n \geq m .\end{cases}
$$

We directly check that $o\left(g_{m}\right)=p^{\alpha_{m}}, x_{m}=g_{m}-p^{\alpha_{m+1}-\alpha_{m}} g_{m+1}$ and $\left\langle\left\{g_{m} / m \geq 1\right\}\right\rangle=\bigoplus_{m \geq 1}\left\langle g_{m}\right\rangle$.

Let $m \in \mathbb{N}^{*}$ and $\xi$ a function from $\mathbb{N}$ into $\{0,1\}$, we define the element $S(m, \xi)$ of $\prod_{n \geq 1}\left\langle x_{n}\right\rangle$ by

$$
\varphi_{n}(S(m, \xi))= \begin{cases}0 & \text { if } n<m \\ \xi(n) t_{n, m} p^{n-m+\alpha_{n-1}} x_{n} & \text { if } n \geq m .\end{cases}
$$

We have

$$
S(m, \xi)=\left(\sum_{n=m}^{r} \xi(n) t_{n, m} p^{n-m+\alpha_{n-1}} x_{n}\right)+t_{r+1, m} p^{r+1-m} S(r+1, \xi)
$$

if $r \geq m$.

Let $K_{1}$ be the subgroup of $\prod_{n \geq 1}\left\langle x_{n}\right\rangle$ generated by $\left\{g_{m} / m \geq 1\right\} \cup\left\{S(m, \xi) / m \geq 1, \xi \in\{0,1\}^{\mathbb{N}}\right\}$. 
Lemma 2.1. The direct sum $\bigoplus_{n \geq 1}\left\langle x_{n}\right\rangle$ is a subgroup of $K_{1}$ and for all $\lambda \in \operatorname{End}\left(K_{1}\right)$ there exist $s, N \in \mathbb{N}$ and $v \in \mathbb{Z}$ such that $t_{s, 1} p^{\alpha_{n}-n} \lambda\left(x_{n}\right)=$ $p^{\alpha_{n}-n} v x_{n}, \forall n \geq N$.

Proof: Let $\lambda \in \operatorname{End}\left(K_{1}\right)$. Let us show at first that there exists $N_{0} \geq 1$ such that if $n>m \geq N_{0}$ then $\varphi_{n}\left(p^{\alpha_{m}-m} \lambda\left(x_{m}\right)\right)=0$.

If not, we can find a sequence $\left(m_{k}\right)_{k \geq 1}$ such that for all $k \geq 1$, there exists $n_{k}>m_{k}$ with $\varphi_{n_{k}}\left(p^{\alpha_{m_{k}}-m_{k}} \lambda\left(x_{m_{k}}\right)\right) \neq 0$ and $\alpha_{n_{k}} \leq m_{k+1}$. Let $\zeta: \mathbb{N} \rightarrow\{0,1\}$ be the function defined by $\zeta(n)=1$ if $n \in\left\{m_{k} / k \geq 1\right\}$ and $\zeta(n)=0$ otherwise. We can write:

$$
\lambda(S(1, \zeta))=\sum_{i=1}^{a} c_{i} g_{i}+\sum_{j=1}^{b} d_{j} S\left(m, \xi_{j}\right) .
$$

If we put $t=\alpha_{a}$, then $p^{t} \lambda(S(1, \zeta))=p^{t} \sum_{j=1}^{b} d_{j} S\left(m, \xi_{j}\right)$. For any $k$, we have

$$
\begin{aligned}
& p^{\theta_{m_{k}}+1} S(1, \zeta)=p^{\theta_{m_{k}}+1} \\
\times & {\left[\left(\sum_{n=1}^{m_{k+1}-1} \zeta(n) t_{n, 1} p^{n-1+\alpha_{n-1}} x_{n}\right)+t_{m_{k+1}, 1} p^{m_{k+1}} S\left(m_{k+1}, \zeta\right)\right] \in p^{\alpha_{n_{k}}} K_{1} }
\end{aligned}
$$

because $\theta_{m_{k}}+1+n-1+\alpha_{n-1} \geq \alpha_{n}$ if $m_{k} \geq n \geq 1, \zeta(n)=0$ if $m_{k+1}>n>m_{k}$ and $\theta_{m_{k}}+1+m_{k+1} \geq \alpha_{n_{k}}$. If $k$ is large enough, then

$$
\varphi_{n_{k}}\left(p^{\theta_{m_{k}}+1} \lambda(S(1, \zeta))\right)=\varphi_{n_{k}}\left(p^{\theta_{m_{k}}+1} \sum_{j=1}^{b} d_{j} S\left(m, \xi_{j}\right)\right)=0
$$

therefore $p^{\theta_{n_{k}}-\theta_{m_{k}}}$ divides $v\left(n_{k}\right)$, where $v(n)=\sum_{j=1}^{b} d_{j} \xi_{j}(n)$. Since the set $\{v(n) / n \in \mathbb{N}\}$ is finite and $\theta_{n_{k}}-\theta_{m_{k}} \geq n_{k}$, then there exists $k_{1} \geq 1$ such that $v\left(n_{k}\right)=0, \forall k \geq k_{1}$. On the other hand

$$
p^{\theta_{m_{k}}-m_{k}+1} S(1, \zeta)-t_{m_{k}, 1} p^{\alpha_{m_{k}}-m_{k}} x_{m_{k}} \in p^{\alpha_{n_{k}}} K_{1},
$$

therefore

$$
\varphi_{n_{k}}\left(p^{\theta_{m_{k}}-m_{k}+1} \sum_{j=1}^{b} d_{j} S\left(m, \xi_{j}\right)\right) \neq 0
$$

for $k$ large enough. Therefore it exists $k_{2} \geq 1$ such that $v\left(n_{k}\right) \neq 0, \forall k \geq k_{2}$, which is absurd. Thus there exists $N_{0} \in \mathbb{N}$ such that: $p^{\alpha_{n}-n} \lambda\left(x_{n}\right)=$ 
$p^{\alpha_{n}-n} r_{n} x_{n}, \forall n \geq N_{0}$, where $r_{n} \in \mathbb{Z}$. Since $T\left(K_{1}\right)=\bigoplus_{m \geq 1}\left\langle g_{m}\right\rangle$ and $\alpha_{k} \leq \alpha_{n}-n$ for $k<n$, therefore $p^{\alpha_{n}-n} \lambda\left(g_{n}\right) \in p^{\alpha_{n}-n}\left(\bigoplus_{k \geq n}\left\langle v g_{k}\right\rangle\right)$. Let $m \geq N_{0}$ and put for $n \geq m, u_{n}=l$ if $p^{\alpha_{n}-m} \lambda\left(g_{n}\right)=p^{\alpha_{n}-m} \sum_{k=n}^{l} t_{k} g_{k}$ with $\left(p^{\alpha_{n}-m} t_{l} g_{l} \neq 0\right.$ and $\left.l>n\right)$ and $u_{n}=0$ if $p^{\alpha_{n}-m} \lambda\left(g_{n}\right) \in p^{\alpha_{n}-m}\left\langle g_{n}\right\rangle$. Since $x_{n}=g_{n}-p^{\alpha_{n+1}-\alpha_{n}} g_{n+1}$, it is easy to see that the sequence $\left(u_{n}\right)_{n \geq m}$ is decreasing. Since for $u_{n} \neq 0$ we have $u_{n}>n$, then there exists $M_{m} \geq m$ such that $u_{n}=0, \forall n \geq M_{m}$. Therefore $p^{\alpha_{n}-m} \lambda\left(g_{n}\right) \in$ $p^{\alpha_{n}-m}\left\langle g_{n}\right\rangle, \forall n \geq M_{m}$. Let $\xi_{0}(n)=1, \forall n \in \mathbb{N}$. We can write: $p^{k^{\prime}} \lambda\left(S\left(1, \xi_{0}\right)\right)=p^{k^{\prime}} \sum_{j=1}^{k} m_{j} S\left(s, \xi_{j}\right)$, where $k^{\prime}, k, s \in \mathbb{N}, m_{1}, \ldots, m_{k} \in \mathbb{Z}$ and $\xi_{1}, \ldots, \xi_{k} \in\{0,1\}^{\mathbb{N}}$.

We have $p^{\theta_{n}-n+1} S\left(1, \xi_{0}\right)-t_{n, 1} p^{\alpha_{n}-n} x_{n} \in p^{\alpha_{n}} K_{1}$ thus for $n$ large enough $t_{n, 1} p^{\alpha_{n}-n} \varphi_{n}\left(\lambda\left(x_{n}\right)\right)=p^{\theta_{n}-n+1} \varphi_{n}\left(\sum_{j=1}^{k} m_{j} S\left(s, \xi_{j}\right)\right) \Longrightarrow p^{n+s-1}$ divides $t_{s, 1} p^{s-1} r_{n}-w(n)$ where $w(n)=\sum_{j=1}^{k} m_{j} \xi_{j}(n)$. Accordingly, if $d \in \mathbb{Z}$ such that the set $\{n \in \mathbb{N} / w(n+1)-w(n)=d\}$ is infinite, then $p^{m}$ divides $d, \forall m \geq N_{0}$, therefore $d=0$. Since the set $\{w(n+1)-w(n) / n \in$ $\mathbb{N}\}$ is finite, then there exist $v_{0} \in \mathbb{Z}$ and $N_{1} \in \mathbb{N}$ such that $w(n)=v_{0}$, $\forall n \geq N_{1}$. It is clear that $p^{s-1}$ divides $v_{0}$. Finally if we put $v_{0}=p^{s-1} v$, we can find $N \in \mathbb{N}$ such that $t_{s, 1} p^{\alpha_{n}-n} \lambda\left(x_{n}\right)=p^{\alpha_{n}-n} v x_{n}, \forall n \geq N$.

Construction 2. Let $\left(\alpha_{n}\right)_{n>0}$ be as in Construction 1, and let $p$ and $q$ be two natural numbers different from zero and relatively prime, we consider the two direct products

$$
\prod_{n \geq 1}\left\langle x_{n}\right\rangle \text { and } \prod_{n \geq 1}\left\langle y_{n}\right\rangle \text { with } o\left(x_{n}\right)=p^{\alpha_{n}} \text { and } o\left(y_{n}\right)=q^{\alpha_{n}}, \quad \forall n \geq 1 .
$$

The elements $h_{m}$ of $\prod_{n \geq 1}\left\langle y_{n}\right\rangle$ are defined in the same way as the $g_{m}$ of $\prod_{n \geq 1}\left\langle x_{n}\right\rangle$ (see Construction 1). The elements $S_{1}(m, \xi)$ (respectively $S_{2}(m, \xi)$ ) of $\prod_{n \geq 1}\left\langle x_{n}\right\rangle$ (respectively $\prod_{n \geq 1}\left\langle y_{n}\right\rangle$ ) are defined like $S(m, \xi)$ of Construction 1 with $t_{n, m}=q^{n-m}\left(\right.$ respectively $\left.t_{n, m}=p^{n-m}\right)$. 
We put $R(m, \xi)=S_{1}(m, \xi)+S_{2}(m, \xi) \in\left(\prod_{n \geq 1}\left\langle x_{n}\right\rangle\right) \oplus\left(\prod_{n \geq 1}\left\langle y_{n}\right\rangle\right)$, then we have,

$R(m, \xi)=\left(\sum_{n=m}^{r} \xi(n)(p q)^{n-m}\left(p^{\alpha_{n-1}} x_{n}+q^{\alpha_{n-1}} y_{n}\right)\right)+(p q)^{r+1-m} R(r+1, \xi)$ if $r \geq m$.

Let $K_{2}$ be the subgroup of $\left(\prod_{n \geq 1}\left\langle x_{n}\right\rangle\right) \oplus\left(\prod_{n \geq 1}\left\langle y_{n}\right\rangle\right)$ generated by $\left\{g_{m} / m \geq 1\right\} \cup\left\{h_{m} / m \geq 1\right\} \cup\left\{R(m, \xi) / m \geq 1, \xi \in\{0,1\}^{\mathbb{N}}\right\}$.

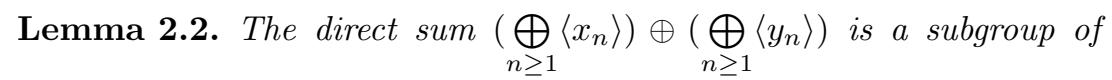
$K_{2}$ and for all $\lambda \in \operatorname{End}\left(K_{2}\right)$, there exist $v \in \mathbb{Z}, N \in \mathbb{N}$ such that $p^{\alpha_{n}-n} \lambda\left(x_{n}\right)=p^{\alpha_{n}-n} v x_{n}$ and $q^{\alpha_{n}-n} \lambda\left(y_{n}\right)=q^{\alpha_{n}-n} v y_{n}, \forall n \geq N$.

Proof: Let $\mu:\left(\prod_{n \geq 1}\left\langle x_{n}\right\rangle\right) \oplus\left(\prod_{n \geq 1}\left\langle y_{n}\right\rangle\right) \rightarrow \prod_{n \geq 1}\left\langle x_{n}\right\rangle$ be the canonical projection. Then $\mu\left(K_{2}\right)$ is the group $K_{1}$ of Construction 1 (with $t_{n, m}=$ $\left.q^{n-m}\right)$. Let $\lambda \in \operatorname{End}\left(K_{2}\right)$. There exists $\lambda_{1} \in \operatorname{End}\left(\mu\left(K_{2}\right)\right)$ such that $\lambda_{1}(\mu(X))=\mu(\lambda(X)), \forall X \in K_{2}$. By Lemma 2.1 there exist $s_{1}, N_{1} \in \mathbb{N}$ and $v_{1} \in \mathbb{Z}$ such that $q^{s_{1}} p^{\alpha_{n}-n} \lambda_{1}\left(x_{n}\right)=p^{\alpha_{n}-n} v_{1} x_{n}, \forall n \geq N_{1}$, therefore $q^{s_{1}} p^{\alpha_{n}-n} \lambda\left(x_{n}\right)=p^{\alpha_{n}-n} v_{1} x_{n}, \forall n \geq N_{1}$. In the same way there are $s_{2}, N_{2} \in \mathbb{N}$ and $v_{2} \in \mathbb{Z}$ such that $p^{s_{2}} q^{\alpha_{n}-n} \lambda\left(y_{n}\right)=q^{\alpha_{n}-n} v_{2} y_{n}, \forall n \geq N_{2}$. We can take $s_{1}=s_{2}=s$ and $N_{1}=N_{2}=N$. Let $\xi_{0}(n)=1, \forall n \in \mathbb{N}$, we can write: $(p q)^{l} \lambda\left(R\left(1, \xi_{0}\right)\right)=(p q)^{l} \sum_{j=1}^{k} m_{j} R\left(m, \xi_{j}\right)$ where $l, k, m \in \mathbb{N}^{*}$, $m_{1}, \ldots, m_{k} \in \mathbb{Z}$ and $\xi_{1}, \ldots, \xi_{k} \in\{0,1\}^{\mathbb{N}}$. We can take $m \geq 1+s$. By applying $\mu$ to this equality, we obtain:

$$
p^{l} \lambda\left(S\left(1, \xi_{0}\right)\right)=p^{l} \sum_{j=1}^{k} m_{j} S\left(m, \xi_{j}\right) .
$$

Then for $n$ large enough $p^{n+m-1}$ divides $q^{m-1-s} p^{m-1} v_{1}-v(n)$ where $v(n)=\sum_{j=1}^{k} m_{j} \xi_{j}(n)$ (see the proof of Lemma 2.1). Let $d \in \mathbb{Z}$ such that the set $\{n \in \mathbb{N} / v(n)=d\}$ is infinite, then $d=q^{m-1-s} p^{m-1} v_{1}$ in the same way $d=p^{m-1-s} q^{m-1} v_{2}$. If we put $v_{1}=q^{s} v$ and $v_{2}=p^{s} v$, then we can find $N \in \mathbb{N}$ such that

$$
p^{\alpha_{n}-n} \lambda\left(x_{n}\right)=p^{\alpha_{n}-n} v x_{n} \text { and } q^{\alpha_{n}-n} \lambda\left(y_{n}\right)=q^{\alpha_{n}-n} v y_{n}, \quad \forall n \geq N .
$$


Construction 3. Let $\left(\alpha_{n}\right)_{n \geq 0}$ be as in Construction 1 and $\left(\beta_{n}\right)_{n \geq 1}$ be a sequence of nonzero natural numbers. Let $p, q_{1}, \ldots, q_{n}, \ldots$ be nonzero relatively prime natural numbers. Let us consider the group $\left(\prod_{n \geq 1}\left\langle x_{n}\right\rangle\right) \oplus$ $\left(\prod_{n \geq 1}\left\langle z_{n}\right\rangle\right)$ with $o\left(x_{n}\right)=p^{\alpha_{n}}$ and $o\left(z_{n}\right)=q_{n}^{\beta_{n}}, \forall n \geq 1$, the elements $g_{m}$ and $S(m, \xi)$ of $\prod_{n \geq 1}\left\langle x_{n}\right\rangle$ are defined as in Construction 1 with

$$
t_{n, m}= \begin{cases}1 & \text { if } n=m \\ q_{1} \cdots q_{m} & \text { if } n=m+1 \\ \left(q_{1} \cdots q_{m}\right)^{n-m}\left(\prod_{j=1}^{n-m-1} q_{m+j}^{n-m-j}\right) & \text { if } n \geq m+2,\end{cases}
$$

the element $R(m, \xi)$ of $\prod_{n \geq 1}\left\langle z_{n}\right\rangle$ is defined as follows

$$
\varphi_{n}(R(m, \xi))= \begin{cases}0 & \text { if } n<m \\ \xi(n) p^{n-m} t_{n, m} z_{n} & \text { if } n \geq m\end{cases}
$$

where $\varphi_{k}: \prod_{n \geq 1}\left\langle z_{n}\right\rangle \rightarrow\left\langle z_{k}\right\rangle$ is the canonical projection. If we put

$$
T(m, \xi)=S(m, \xi)+R(m, \xi) \in\left(\prod_{n \geq 1}\left\langle x_{n}\right\rangle\right) \oplus\left(\prod_{n \geq 1}\left\langle z_{n}\right\rangle\right),
$$

we have

$T(m, \xi)=\left(\sum_{n=m}^{r} \xi(n) t_{n, m} p^{n-m}\left(p^{\alpha_{n-1}} x_{n}+z_{n}\right)\right)+t_{r+1, m} p^{r+1-m} T(r+1, \xi)$, if $r \geq m$.

Let $K_{3}$ be the subgroup of $\left(\prod_{n \geq 1}\left\langle x_{n}\right\rangle\right) \oplus\left(\prod_{n \geq 1}\left\langle z_{n}\right\rangle\right)$ generated by $\left\{g_{n} / n \geq\right.$ 1\} $\cup\left\{z_{n} / n \geq 1\right\} \cup\left\{T(m, \xi) / m \geq 1, \xi \in\{0,1\}^{\mathbb{N}}\right\}$.

Lemma 2.3. The direct sum $\left.\left(\bigoplus_{n \geq 1}\left\langle x_{n}\right\rangle\right) \oplus \underset{n \geq 1}{\bigoplus}\left\langle z_{n}\right\rangle\right)$ is a subgroup of $K_{3}$ and for all $\lambda \in \operatorname{End}\left(K_{3}\right)$, there exist $v \in \mathbb{Z}$ and $N, s \in \mathbb{N}$ such that $t_{s, 1} p^{\alpha_{n}-n} \lambda\left(x_{n}\right)=p^{\alpha_{n}-n} v x_{n}$ and $t_{s, 1} \lambda\left(z_{n}\right)=v z_{n}, \forall n \geq N$.

Proof: Let $\mu:\left(\prod_{n \geq 1}\left\langle x_{n}\right\rangle\right) \oplus\left(\prod_{n \geq 1}\left\langle z_{n}\right\rangle\right) \rightarrow \prod_{n \geq 1}\left\langle x_{n}\right\rangle$ be the canonical projection. Then $\mu\left(K_{3}\right)=K_{1}$ is the group of Construction 1. Let $\lambda \in$ $\operatorname{End}\left(K_{3}\right)$, the endomorphism $\lambda_{1}$ of $K_{1}$ defined by $\lambda_{1}(\mu(X))=\mu(\lambda(X))$, 
$\forall X \in K_{3}$, is well defined. According to Lemma 2.1 there exist $s, N_{0} \in \mathbb{N}$ and $v \in \mathbb{Z}$ such that $t_{s, 1} p^{\alpha_{n}-n} \lambda_{1}\left(x_{n}\right)=p^{\alpha_{n}-n} v x_{n}, \forall n \geq N_{0}$. It is clear that $\lambda\left(z_{n}\right) \in\left\langle z_{n}\right\rangle, \forall n \geq 1$. Putting $\lambda\left(z_{n}\right)=k_{n} z_{n}, \forall n \geq 1$, we consider $\xi_{0}: \mathbb{N} \rightarrow\{0,1\}$ with $\xi_{0}(n)=1, \forall n \in \mathbb{N}$ we can write: $p^{l} r \lambda\left(T\left(1, \xi_{0}\right)\right)=p^{l} r \sum_{j=1}^{k} d_{j} T\left(m, \xi_{j}\right)$ where $r$ and $p$ are relatively prime and $m \geq s$. By applying $\mu$ to this equality, we obtain:

$$
p^{l} \lambda_{1}\left(S\left(1, \xi_{0}\right)\right)=p^{l} \sum_{j=1}^{k} d_{j} S\left(m, \xi_{j}\right) .
$$

Following the same steps as in Lemmas 2.1 and 2.2, we can find $N_{1} \in \mathbb{N}$ such that $p^{n+m-1}$ divides $t_{m, s} p^{m-1} v-v(n), \forall n \geq N_{1}$, with $v(n)=$ $\sum_{j=1}^{k} d_{j} \xi_{j}(n)$. Then there exists $N_{2}$ such that $v(n)=t_{m, s} p^{m-1} v, \forall n \geq N_{2}$. If $n \geq m$, then $q_{n}^{\beta_{n}}$ divides $t_{m, 1} p^{m-1} k_{n}-v(n)$. Finally there exists $N \in \mathbb{N}$ such that $t_{s, 1} p^{\alpha_{n}-n} \lambda\left(x_{n}\right)=v p^{\alpha_{n}-n} x_{n}$ and $t_{s, 1} \lambda\left(z_{n}\right)=v z_{n}, \forall n \geq N$.

\section{Characterization of the inessential endomorphisms in the category of the Abelian groups}

In the following, we suppose that $A$ is a group, and $f$ an endomorphism of $A$ satisfying the following property.

$(E)$ : For any exact sequence $0 \rightarrow A \stackrel{\sigma}{\rightarrow} B$ there exists $\tilde{f} \in \operatorname{End}(B)$ such that the following diagram

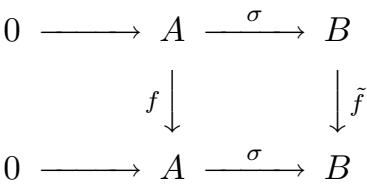

is commutative.

Let $\left(\alpha_{n}\right)_{n \geq 0}$ be a sequence as in Construction 1 .

Lemma 3.1. For all $a \in A$ and any $q \in \mathbb{N}^{*}$, there exists $v \in \mathbb{Z}$ such that $(f(a)-v a) \in \bigcap_{n \geq 0} q^{n} A$.

Proof: Let us consider the free group $L=\bigoplus_{n \geq 1}\left\langle e_{n}\right\rangle$. We put $G=A \oplus L$, $G_{0}=\left\langle\left\{a-q^{\alpha_{n}} e_{n} / n \geq 1\right\}\right\rangle$ and $\bar{G}=G / G_{0}$. The homomorphism $\sigma: A \rightarrow$ $\bar{G}$ defined by $\sigma(b)=b+G_{0}$ is a monomorphism, and if $x_{n}=\overline{e_{n}}+\sigma(A)$ $\left(\overline{e_{n}}=e_{n}+G_{0}\right)$ then $\bar{G} / \sigma(A)=\bigoplus_{n \geq 1}\left\langle x_{n}\right\rangle$ and $o\left(x_{n}\right)=q^{\alpha_{n}}, \forall n \geq 1$. Let 
$K_{1}$ be a subgroup of $\prod_{n \geq 1}\left\langle x_{n}\right\rangle$ defined in Construction 1 (with $t_{n, m}=1$, $\forall n \geq m$ ). There exists a commutative diagram, whose rows are exact, and which has the following form:

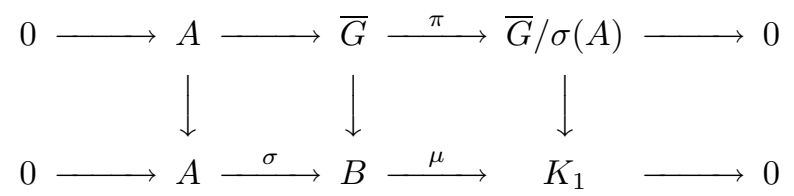

(see $[3,24.6])$. We can find $\tilde{f} \in \operatorname{End}(B)$ and $\lambda \in \operatorname{End}\left(K_{1}\right)$ such that $\tilde{f} \sigma=\sigma f$ and $\lambda \mu=\mu \tilde{f}$. By Lemma 2.1, there are $v \in \mathbb{Z}$ and $N \in \mathbb{N}$ such that $q^{\alpha_{n}-n} \lambda\left(x_{n}\right)=v q^{\alpha_{n}-n} x_{n}, \forall n \geq N$. For $n \geq N, \mu\left[q^{\alpha_{n}-n}\left(\tilde{f}\left(\overline{e_{n}}\right)-\right.\right.$ $\left.\left.v e_{n}\right)\right]=0$, therefore $(f(a)-v a) \in q^{n} A$, so $(f(a)-v a) \in \bigcap_{n \geq 0} q^{n} A$.

Corollary 3.2. If $A^{1}=0$, then for all $a \in T(A)$ there exists $v_{a} \in \mathbb{Z}$ such that $f(a)=v_{a} a$ where $T(A)$ is the torsion part of $A$.

Proof: Let us put $q=o(a)$ and let $v \in \mathbb{Z}$ such that $(f(a)-v a) \in \bigcap_{n \geq 0} q^{n} A$. Let $p$ be a prime number, if $p$ divides $q$ then $(f(a)-v a) \in \bigcap_{n \geq 0} p^{n} A$ and if $p$ and $q$ are relatively prime, we also have $(f(a)-v a) \in \bigcap_{n \geq 0} p^{n} A$, thus $f(a)=v a$.

Lemma 3.3. If $A^{1}=0$, then there exists $v \in \mathbb{Z}$ such that $f(a)=v a$, $\forall a \in T(A)$.

Proof: We suppose that $T(A)$ is bounded, then there exists $x_{0} \in T(A)$ such that $\left\langle x_{0}\right\rangle$ is a direct summand of $T(A)$ and $o\left(x_{0}\right) . T(A)=0$. If $f\left(x_{0}\right)=v x_{0}$, then $\forall a \in T(A), f(a)=v a$. We now suppose that $T(A)$ is not bounded. If $p$ is prime number, we denote by $T_{p}$ the $p$-component of $T(A)$.

1st case: There exists a prime number $p$ such that $T_{p}$ is not bounded. Let $S$ be a basic subgroup of $T_{p}$, we can write

$$
S=\left(\bigoplus_{n \geq 1}\left\langle a_{n}\right\rangle\right) \oplus S_{0} \text { with } o\left(a_{n}\right)=p^{r_{n}} \text { and } 1 \leq r_{n}<r_{n+1}, \quad \forall n \geq 1 .
$$


For each $n \geq 1$, we consider $a_{n}$ as an element of the group $\left\langle X_{n}\right\rangle$ with $p^{\alpha_{n}} X_{n}=a_{n}$. There exists a group $G$ such that:

$$
\begin{aligned}
A & \leq G, \\
\left(\bigoplus_{n \geq 1}\left\langle X_{n}\right\rangle\right) & \leq G, \\
A+\left(\bigoplus_{n \geq 1}\left\langle X_{n}\right\rangle\right) & =G
\end{aligned}
$$

and

$$
A \cap\left(\bigoplus_{n \geq 1}\left\langle X_{n}\right\rangle\right)=\bigoplus_{n \geq 1}\left\langle a_{n}\right\rangle .
$$

We put $x_{n}=X_{n}+A$, then $G / A=\bigoplus_{n \geq 1}\left\langle x_{n}\right\rangle$ and $o\left(x_{n}\right)=p^{\alpha_{n}}, \forall n \geq 1$.

By [3, Proposition 24.6], there exists a commutative diagram, whose rows are exact, and has the following form:

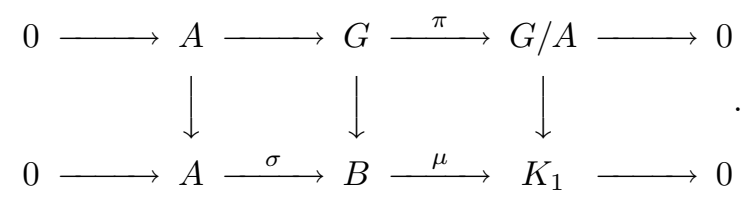

$K_{1}$ is the group of Construction 1 (with $t_{n, m}=1, \forall n \geq m$ ). There are $\tilde{f} \in \operatorname{End}(B)$ and $\lambda \in \operatorname{End}\left(K_{1}\right)$ such that $\tilde{f} \sigma=\sigma f$ and $\lambda \mu=\mu \tilde{f}$. There exist $v \in \mathbb{Z}$ and $N \in \mathbb{N}$ such that $p^{\alpha_{n}-n} \lambda\left(x_{n}\right)=v p^{\alpha_{n}-n} x_{n}, \forall n \geq N$ (Lemma 2.1). We have for each $n \geq N, \mu\left[p^{\alpha_{n}-n}\left(\tilde{f}\left(X_{n}\right)-v X_{n}\right)\right]=0$, so that $\left(f\left(a_{n}\right)-v a_{n}\right) \in p^{n} A$.

Let us put $f\left(a_{n}\right)=k_{n} a_{n}$ (Corollary 3.2), then we have $p^{n}$ divides $k_{n}-v, \forall n \geq N$. By using again Corollary 3.2, we can establish easily that $f\left(a_{n}\right)=v a_{n}, \forall n \geq 1$. Let $b \in T_{q}$ with $q \neq p$, and put $o(b)=q^{s}$. Let us consider the free group $L=\bigoplus_{n \geq 0}\left\langle e_{n}\right\rangle$. Let $L_{0}$ be the subgroup of $L$ generated by $\left\{q^{s} e_{0}\right\} \cup\left\{q^{\alpha_{n}} e_{n}-e_{0} / n \geq 1\right\}$. 
We consider $b$ as an element of $\bar{L}=L / L_{0}$ by identifying $b$ with $\overline{e_{0}}=$ $e_{0}+L_{0}$. There exists a group $G_{1}$ such that $A \leq G_{1}$,

$$
\begin{array}{r}
\left(\bigoplus_{n \geq 1}\left\langle X_{n}\right\rangle\right) \oplus \bar{L} \leq G_{1}, \\
A+\left(\left(\bigoplus_{n \geq 1}\left\langle X_{n}\right\rangle\right) \oplus \bar{L}\right)=G_{1}
\end{array}
$$

and

$$
A \cap\left(\left(\bigoplus_{n \geq 1}\left\langle X_{n}\right\rangle\right) \oplus \bar{L}\right)=\left(\bigoplus_{n \geq 1}\left\langle a_{n}\right\rangle\right) \oplus\langle b\rangle .
$$

We put $x_{n}^{\prime}=X_{n}+A$ and $y_{n}=\overline{e_{n}}+A$, then $o\left(x_{n}^{\prime}\right)=p^{\alpha_{n}}, o\left(y_{n}\right)=q^{\alpha_{n}}$. $\forall n \geq 1$, and $G_{1} / A=\left(\bigoplus_{n \geq 1}\left\langle x_{n}\right\rangle\right) \oplus\left(\bigoplus_{n \geq 1}\left\langle y_{n}\right\rangle\right)$.

Let $K_{2}$ be the group of Construction 2, there exists a commutative diagram, whose rows are exact, and has the following form:

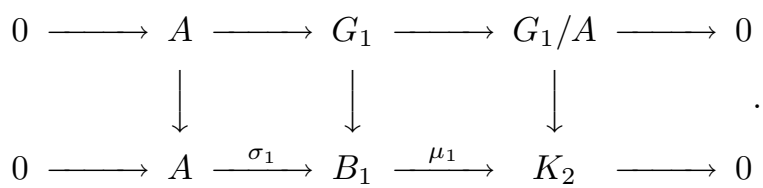

There exist $\tilde{f}_{1} \in \operatorname{End}\left(B_{1}\right)$ and $\lambda_{1} \in \operatorname{End}\left(K_{2}\right)$ such that $\tilde{f}_{1} \sigma_{1}=\sigma_{1} f$ and $\lambda_{1} \mu_{1}=\mu_{1} \tilde{f}_{1}$. By Lemma 2.2, there exist $k \in \mathbb{Z}$ and $M \in \mathbb{N}$ such that $p^{\alpha_{n}-n} \lambda_{1}\left(x_{n}^{\prime}\right)=k p^{\alpha_{n}-n} x_{n}^{\prime}$ and $q^{\alpha_{n}-n} \lambda_{1}\left(y_{n}\right)=k q^{\alpha_{n}-n} y_{n}, \forall n \geq M$. For $n \geq M, p^{\alpha_{n}-n} \mu_{1}\left(\tilde{f}\left(X_{n}\right)-k X_{n}\right)=0$ and $q^{\alpha_{n}-n} \mu_{1}\left(\tilde{f}\left(\overline{e_{n}}\right)-k \overline{e_{n}}\right)=0$ and so $\left(f\left(a_{n}\right)-k a_{n}\right) \in p^{n} A$ and $(f(b)-k b) \in q^{n} A$. Then $k=v$ and $f(b)=v b$. Therefore it is easy to deduce that $f(a)=v a, \forall a \in T(A)$.

2nd case: $T_{p}$ is bounded for any prime number $p$. We can write $T(A)=$ $\bigoplus_{n \geq 1} T_{p_{n}}$ and let for each $n \geq 1, b_{n} \in T_{p_{n}}$ such that $\left\langle b_{n}\right\rangle$ is a direct summand of $T_{p_{n}}$ and $o\left(b_{n}\right) T_{p_{n}}=0$.

We put $o\left(b_{n}\right)=p_{n}^{\beta_{n}}$ and we consider $b_{n}$ as an element of the group $\left\langle Z_{n}\right\rangle$ with $p_{n}^{\beta_{n}} Z_{n}=b_{n}$. We take $m \geq 1$, there exists a group $H$ such that $A \leq H,\left(\bigoplus_{n \geq m}\left\langle Z_{n}\right\rangle\right) \leq H, H=A+\left(\bigoplus_{n \geq m}\left\langle Z_{n}\right\rangle\right)$ and $A \cap\left(\bigoplus_{n \geq m}\left\langle Z_{n}\right\rangle\right)=$ $\bigoplus_{n \geq m}\left\langle b_{n}\right\rangle$. If $z_{n}=Z_{n}+A$, then $H / A=\bigoplus_{n \geq m}\left\langle z_{n}\right\rangle$. By using Lemma 2.3 and [3, Proposition 24.6], as before, we can find $r_{1} \in \mathbb{N}^{*}$ whose only 
prime factors are $p_{m}, \ldots, p_{m^{\prime}}, m^{\prime} \geq m$ and $\left(v_{1}, N_{1}\right) \in \mathbb{Z} \times \mathbb{N}$ such that $r_{1} f\left(b_{n}\right)=v_{1} b_{n}, \forall n \geq N_{1}$. in the same way there exists $r_{2} \in \mathbb{N}$ whose only prime factors are $p_{m^{\prime}+1}, \ldots, p_{m^{\prime \prime}}$ (in particular $r_{2} \wedge r_{1}=1$ ) and $\left(v_{2}, N_{2}\right) \in \mathbb{Z} \times \mathbb{N}$ such that $r_{2} f\left(b_{n}\right)=v_{2} b_{n}, \forall n \geq N_{2}$. If $v=\gamma_{1} v_{1}+\gamma_{2} v_{2}$ (where $\gamma_{1} r_{1}+\gamma_{2} r_{2}=1$ ) then for $N=\sup \left(N_{1}, N_{2}\right)$ we have $f\left(b_{n}\right)=v b_{n}$, $\forall n \geq N$.

We now suppose $n_{1}<N$ and put $p=p_{n_{1}}, \beta=\beta_{n_{1}}$. Let $L=\bigoplus_{n \geq 0}\left\langle e_{n}\right\rangle$ be the free group and $\bar{L}=L / L_{1}$ where $L_{1}=\left\langle\left\{p^{\beta} e_{0}\right\} \cup\left\{p^{\alpha_{n}} e_{n}-e_{0} / n \geq\right.\right.$ $1\}\rangle$ there exists a group $H_{1}$ such that $A \leq H_{1}, \bar{L} \oplus\left(\bigoplus_{n \geq N}\left\langle Z_{n}\right\rangle\right) \leq H_{1}$, $A+\left(\bar{L} \oplus\left(\bigoplus_{n \geq N}\left\langle Z_{n}\right\rangle\right)\right)=H_{1}$ and $A \cap\left(\bar{L} \oplus\left(\bigoplus_{n \geq N}\left\langle Z_{n}\right\rangle\right)\right)=\left\langle b_{n_{1}}\right\rangle \oplus\left(\bigoplus_{n \geq N}\left\langle b_{n}\right\rangle\right)$.

Now put $x_{n}=\overline{e_{n}}+A\left(\overline{e_{n}}=e_{n}+\bar{L}_{1}\right)$ and $z_{n}=Z_{n}+A$, then $o\left(x_{n}\right)=p^{\alpha_{n}}, o\left(z_{n}\right)=p_{n}^{\beta_{n}}$ and

$$
H_{1} / A=\left(\bigoplus_{n \geq 1}\left\langle x_{n}\right\rangle\right) \oplus\left(\bigoplus_{n \geq 1}\left\langle y_{n}\right\rangle\right) .
$$

By applying again Lemma 2.3 and [3, Proposition 24.6] we show that $f\left(a_{n_{1}}\right)=v a_{n_{1}}$. Thus $f\left(a_{n}\right)=v a_{n}, \forall n \geq 1$ and thereafter $f(a)=v a$, $\forall a \in T(A)$.

Lemma 3.4. If $A^{1}=0$ and $T(A)=0$, then there exists $v \in \mathbb{Z}$ such that $f=v \operatorname{id}_{A}$.

Proof: Let $a \in A$ with $a \neq 0$. There exists a prime number $p$ such that $a \notin \bigcap_{n \geq 0} p^{n} A$. According to Lemma 3.1 there exists $v \in \mathbb{Z}$ such that $(f(a)-v a) \in \bigcap_{n \geq 0} p^{n} A$. Let $q \in \mathbb{N}^{*}$, there exists $v_{q} \in \mathbb{Z}$ such that $\left(f(a)-v_{q} a\right) \in \bigcap_{n \geq 0}(p \bar{q})^{n} A$.

We have $\left(v-v_{q}\right) a \in \bigcap_{n \geq 0} p^{n} A$, this implies $v_{q}=v$, and thereafter $f(a)=v a$. Since $A$ is torsion-free, it is easy to establish that $f(b)=v b$, $\forall b \in A$.

Lemma 3.5. If $A^{1}=0$, then there exists $v \in \mathbb{Z}$ such that $f=v \operatorname{id}_{A}$.

Proof: By Lemma 3.3, there exists $v \in \mathbb{Z}$ such that $f(x)=v x, \forall x \in$ $T(A)$.

Let $a \in A$, we will show that $f(a) \in\langle a\rangle$. We Suppose that $(f(a)-$ $v a) \neq 0$, then there exists a prime number $p$ such that $(f(a)-v a) \notin$ 
$\bigcap_{n \geq 0} p^{n} A$. By Lemma 3.1, there exists $r \in \mathbb{Z}$ such that $(f(a)-r a) \in$ $\bigcap_{\geq}^{n} p^{n} A$, there also exists for all $q \in \mathbb{N}^{*}$ an $r_{q} \in \mathbb{Z}$ such that $\left(f(a)-r_{q} a\right) \in$ $n \geq 0$

$\bigcap(p q)^{n} A$. Assume $r_{q} \neq r$ for some number $q$. $n \geq 0$

Since $\left(r-r_{q}\right) a \in \bigcap_{n \geq 0} p^{n} A$, then there exists $s \in \mathbb{N}$ such that $p^{s} a \in$ $\bigcap_{n \geq 0} p^{n} A$. Therefore $\forall n \in \mathbb{N}$, there exists $a_{n} \in A$ such that $p^{s}\left(a-p^{n} a_{n}\right)=$ 0 , it follows that $f\left(a-p^{n} a_{n}\right)=v\left(a-p^{n} a_{n}\right)$ and hence $(f(a)-v a) \in p^{n} A$, which is absurd. Thus $r_{q}=r, \forall q \in \mathbb{N}^{*}$ and thereafter $f(a)=r a$.

Now, we will distinguish two cases:

1st case: $T(A)$ is not bounded. Let $a \in A$ with $o(a)=\infty$ and put $f(a)=r a . \forall x \in T(A), f(a+x)=r^{\prime}(a+x)=r a+v x$ which implies that $r=r^{\prime}$ and $\left(v-r^{\prime}\right) x=0$, since $T(A)$ is not bounded so $r=v$.

2nd case: $T(A)$ is bounded, let $m \in \mathbb{N}^{*}$ such that $m T(A)=0$.

We consider the exact sequence $0 \rightarrow T(A) \rightarrow A \rightarrow m A \rightarrow 0$. By [3, Proposition 24.6] it is easy to see that the endomorphism $g$ of $m A$ defined by $g(m a)=m f(a)$ satisfies the property $(E)$, since $T(m A)=0$ and $(m A)^{1}=0$ then according to Lemma 3.4 there exists $r \in \mathbb{Z}$ such that $m f(a)=r m a, \forall a \in A$.

We suppose $T(A) \neq A$. Let $a \in A$ with $o(a)=\infty$ and $f(a)=r_{a} a$, therefore $m\left(r_{a}-a\right) a=0$ and hence $r_{a}=r$.

Let $x \in T(A)$, then $f(a+x)=r(a+x)=r a+v x$ which implies that $(r-v) x=0$, thus $f(x)=r x$.

Finally $\forall b \in A, f(b)=r b$.

Theorem 3.6. If $A$ is reduced, then there exists $v \in \mathbb{Z}$ such that $f=$ $v \operatorname{id}_{A}$.

Proof: Let $x \in A$ such that $\langle x\rangle$ is a direct summand of $A$.

We can write $A=\langle x\rangle \oplus A_{0}$. Let $S$ be a divisible group such that $x \in S$.

Let $\sigma: A \rightarrow S \oplus A_{0}, \sigma\left(n x+a_{0}\right)=n x+a_{0}$. Then there exists $\tilde{f} \in$ $\operatorname{End}\left(S \oplus A_{0}\right)$ such that $\tilde{f} \sigma=\sigma f$. If we put $f(x)=m x+a_{0}$ with $m \in \mathbb{Z}$ and $a_{0} \in A_{0}$, we get $a_{0}=\tilde{f}(x)-m x \in S \cap A_{0}=0$ which implies that $a_{0}=0$ and thereafter $f(x)=m x$. Therefore if $\langle x\rangle$ is a direct summand of $A$, then $f(x) \in\langle x\rangle$.

By Lemma 3.5 there exists $v \in \mathbb{Z}$ such that $(f-v$ id $)(A) \subseteq A^{1}$. We put $\rho=f-v \operatorname{id}_{A}$. 
Show first that $\rho(T(A))=0$. Let $B$ be $p$-basic subgroup of $T(A)$ ( $p$ is a prime number), $B=\bigoplus_{i \in I}\left\langle x_{i}\right\rangle$ and $\forall i \in I,\left\langle x_{i}\right\rangle$ is a direct summand of $A$. We put for $i \in I, f\left(x_{i}\right)=m_{i} x_{i}$. We have $\left(m_{i}-v\right) x_{i} \in A^{1}$ which implies that $m_{i} x_{i}=v x_{i}=f\left(x_{i}\right)$.

Then $\rho(B)=0$ and thereafter $\rho(T(A))$ is $p$ divisible. Therefore, $\rho(T(A))$ is divisible and so $\rho(T(A))=0$. Let us put $A / T(A)=(D / T(A)) \oplus$ $(R / T(A))$ with $D / T(A)$ divisible, $R / T(A)$ reduced, $T(A) \leq D$ and $A^{1} \leq D$.

The homomorphism $\bar{\rho}: A / T(A) \rightarrow A$ where $\bar{\rho}(a+T(A))=\rho(a)$ is well defined and $\bar{\rho}(D / T(A))=\rho(D)=0$ because $D / T(A)$ is divisible and $A$ is reduced. There exists a torsion-free divisible group $C$ such that $A / D \leq C$. By [3, Proposition 24.6], there exists a commutative diagram, whose rows are exact, and has the following form:

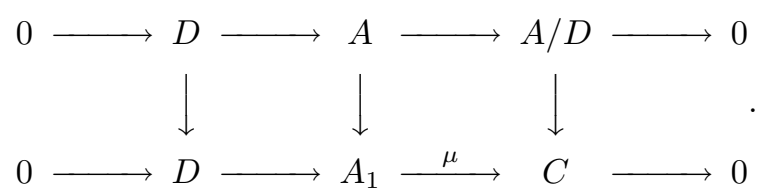

Let $D_{1}$ be the maximal divisible subgroup of $A_{1}, D \cap D_{1}$ is then divisible.

In fact if $x \in D \cap D_{1}$ and $n \in \mathbb{N}^{*}$, we can write $x=n y$ with $y \in D_{1}$ and $\mu(x)=n \mu(y)=0$ so $\mu(y)=0$ because $C$ is torsion-free, therefore $y \in D$. Since $A$ is reduced, then $D \cap D_{1}=0$. there exists $f_{1} \in \operatorname{End}\left(A_{1}\right)$ such that $f_{1}(a)=f(a), \forall a \in A$.

If we put $\rho_{1}=f_{1}-v \operatorname{id}_{A_{1}}$, we have $\rho_{1}(A)=\rho(A) \subseteq A^{1} \subseteq D$, from an other side the homomorphism $\overline{\rho_{1}}: A_{1} / D \rightarrow A_{1}$ such that $\overline{\rho_{1}}\left(a_{1}+\right.$ $D)=\rho_{1}\left(a_{1}\right)$, for $a_{1} \in A_{1}$, is well defined. Thus $\rho_{1}\left(A_{1}\right)$ is divisible and thereafter $\rho_{1}\left(A_{1}\right) \subseteq D_{1}$. We then conclude that $\rho(A) \subseteq D \cap D_{1}=0$ which implies that $\rho=0$.

Corollary 3.7. Let $A$ be a group and $f$ be an endomorphism of $A, f$ satisfies $(E)$ if and only if there exists $v \in \mathbb{Z}$ such that $\left(f-v \operatorname{id}_{A}\right)(A) \subseteq D$, where $D$ is maximal divisible subgroup of $A$.

Proof: According to [3, Proposition 24.6], the endomorphism $\bar{f}$ of $\bar{A}=$ $A / D,(\bar{f}(\bar{a})=\overline{f(a)})$ satisfies $(E)$. By Theorem 3.6, there exists $v \in \mathbb{Z}$ such that $\left(f-v \operatorname{id}_{A}\right)(A) \subseteq D$.

The second assertion is easy to establish.

We end this paper by the following remarks: 
1. Let $C$ be a reduced group. $C$ is rigid (according to the terminology of [2]) if and only if $C$ is torsion cyclic or $C$ is torsion-free and $\operatorname{End}(C) \cong \mathbb{Z}$.

2. A group $A$ is rigid if and only if $A=D \oplus C$ with $D$ divisible and $C$ reduced rigid.

3. For any cardinal $m$ there exists a rigid group of cardinality $m([\mathbf{1}]$, $[4]$ and $[5])$.

Acknowledgements. The authors are indebted to the referee for the very useful comments on earlier version of this article and for providing additional references.

\title{
References
}

[1] A. L. S. Corner And R. GÖBel, Prescribing endomorphism algebras, a unified treatment, Proc. London Math. Soc. (3) 50(3) (1985), 447-479.

[2] M. Dugas And R. GöBel, Applications of abelian groups and model theory to algebraic structures, in: "Infinite groups" (Ravello, 1994), de Gruyter, Berlin, 1996, pp. 41-62.

[3] L. Fuchs, "Infinite abelian groups", Vol. I, Pure and Applied Mathematics 36, Academic Press, New York-London, 1970.

[4] L. Fuchs, "Infinite abelian groups", Vol. II, Pure and Applied Mathematics 36-II, Academic Press, New York-London, 1973.

[5] R. GÖBEL AND W. MAY, Four submodules suffice for realizing algebras over commutative rings, J. Pure Appl. Algebra 65(1) (1990), 29-43.

\author{
Department of Mathematics and Computer Science \\ Faculty of Science \\ Mohammed V University \\ B. P. 1014 \\ Rabat \\ Morocco \\ E-mail address: seddikabd@hotmail.com \\ E-mail address: esanouni@challa.fsr.ac.ma
}

Primera versió rebuda el 21 de juny de 2002,

darrera versió rebuda el 4 d'octubre de 2002. 